\title{
Exploring insertions and deletions (indels) of MSRB3 gene and their association with growth traits in four Chinese indigenous cattle breeds
}

\author{
Mingli $\mathrm{Wu}^{1}$, Shipeng $\mathrm{Li}^{1}$, Guoliang Zhang ${ }^{2}$, Yingzhi Fan ${ }^{1}$, Yuan Gao ${ }^{1}$, Yongzhen Huang ${ }^{1}$, \\ Xianyong Lan $^{1}$, Chuzhao Lei ${ }^{1}$, Yun Ma ${ }^{3,4}$, and Ruihua Dang ${ }^{1}$ \\ ${ }^{1}$ Key Laboratory of Animal Genetics, Breeding and Reproduction of Shaanxi Province, College of Animal \\ Science and Technology, Northwest A\&F University, Yangling, Shaanxi, 712100, P. R. China \\ ${ }^{2}$ Branch of Animal Husbandry, Jilin Academy of Agricultural Science, Gongzhuling, Jilin, 136100, P. R. China \\ ${ }^{3}$ College of Life Sciences, Xinyang Normal University, Xinyang, Henan, 464000, P. R. China \\ ${ }^{4}$ School of Agriculture, Ningxia University, Yinchuan, Ningxia, 750021, P. R. China
}

Correspondence: Ruihua Dang (dangruihua@nwsuaf.edu.cn) and Yun Ma (tmlf74@ 126.com)

Received: 2 October 2018 - Revised: 10 April 2019 - Accepted: 21 June 2019 - Published: 25 July 2019

\begin{abstract}
Methionine sulfoxide reductase B3 (MSRB3) is instrumental in ossification and fat deposition, which regulate the growth and development of cattle directly. The purpose of this study was aimed to explore insertions and deletions (indels) in MSRB3 gene and investigate their association with growth traits in four indigenous cattle breeds (Luxi cattle, Qinchuan cattle, Nanyang cattle, and Jiaxian Red cattle). Four indels were identified by sequencing with DNA pool. Association analysis showed that three of them were associated with growth traits $(P<0.05)$. For P1, the DD (deletion and deletion) genotype was significantly associated with body length of Nanyang cattle; for P6, II (insertion and insertion) and/or DD genotypes were significantly associated with enhanced growth traits of Qinchuan cattle; for P7, II genotype was significantly associated with hucklebone width of Luxi cattle. Our results demonstrated that the polymorphisms in bovine MSRB3 gene were significantly associated with growth traits, which could be candidate loci for marker-assisted selection (MAS) in cattle breeding.
\end{abstract}

\section{Introduction}

There are abundant indigenous cattle breeds in China and most of them were draft cattle for thousands of years (Zhang et al., 2018). Chinese indigenous cattle breeds have the characteristics of slow growth, low yield, and low-grade beef (Yue et al., 2019). With the rapid development of livestock husbandry, beef cattle improvement has become a heated and difficult problem (Singh et al., 2011). However, because of the slow progress in traditional breeding, we need to seek a more efficient and accurate way of breeding urgently (Gui et al., 2015). A noteworthy method is molecular breeding technology, which emerged and developed into an indispensable breeding method, and indels is one of the genetic markers used for assisted selection (Zhang et al., 2003; Fan et al., 2007).
The identification of candidate genes for growth traits has attracted more and more attention. MSRB3 (methionine sulfoxide reductase B3) is an essential member of the $M S R B$ gene family, which can reduce the catalysis effect of methionine-R-sulfoxide to methionine specifically as an oxidoreductase (Kwak et al., 2017a). Previous research has shown that MSRB3 gene could influence ear shape and size, otitis media, heat resistance, oxidation resistance, auditory system, reproductive traits, and size of the hippocampus (Zhang et al., 2017; Fan et al., 2007; Pillas et al., 2010; Shen et al., 2015; Wei et al., 2015; Lee et al., 2012). In recent years, based on the analysis of GWASs (genome-wide association studies), gene silencing, and protein precipitation, MSRB3 was regarded as a candidate gene to affect bovine growth traits (Saatchi et al., 2014). At the same time, it inhibited the proliferation of mouse embryonic fibroblasts and 
human dermal fibroblasts by acting on the p53, p21, and p27 pathways (Lee et al., 2014; Kwak et al., 2017b).

Consequently, the purpose of this research is to obtain mutation information on MSRB3 gene and its association with the body size of Chinese indigenous cattle through the indel screening and association analysis with growth traits.

\section{Materials and methods}

All experiments performed in this study were approved by the International Animal Care and Use Committee of the Northwest A\&F University (IACUC-NWAFU). Furthermore, the care and use of animals was fully compliant with local animal welfare laws, guidelines, and policies.

\subsection{DNA samples and data collection}

A total of 673 Chinese indigenous cattle were used in this study, including Luxi cattle (LX, $N=113$, Shandong Province), Qinchuan cattle (QC, $N=286$, Shaanxi Province), Nanyang cattle (NY, $N=135$, Henan Province), and Jiaxian Red cattle (JX, $N=139$, Henan Province) (Huang et al., 2017, 2018). All individuals selected were between 2 and 5 years old, healthy, and unrelated. Blood samples were collected in anticoagulant blood vessel and stored at $-80^{\circ} \mathrm{C}$ (Zhao et al., 2017a). In order to demonstrate the relationship between polymorphisms and performance traits, more than 6000 growth traits were measured and calculated, including body weight (BW), body height $(\mathrm{BH})$, body length (BL), chest circumference (ChC), chest depth (ChD), chest width $(\mathrm{ChW})$, hucklebone width $(\mathrm{HuW})$, hip width (HW), cannon circumference $(\mathrm{CaC})$, body length index (BLI), chest circumference index (ChCI), chest width index (ChWI), cannon circumference index $(\mathrm{CaCI})$, hucklebone width index (HuWI), and trunk index (TI) (Zhang et al., 2019; Zhao et al., 2018). All data were measured by the same person following the same standard (Zhao et al., 2018).

\subsection{DNA isolation and genomic DNA pools construction}

Genomic DNA was isolated from leukocytes and the method refers to a whole-blood genomic DNA extraction kit (Aidlab Biotechnologies Co., Ltd., China). DNA quantity and purity $\left(\mathrm{OD}_{260} / \mathrm{OD}_{280}\right)$ for each sample was as-

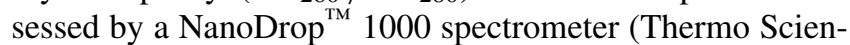
tific, Waltham, MA, USA). DNA samples were diluted to a standard concentration $\left(50 \mathrm{ng} \mu \mathrm{L}^{-1}\right)$ and stored at $-80^{\circ} \mathrm{C}$. To explore the allele variation of the bovine MSRB3 gene, every 25 samples were mixed and used for polymerase chain reaction (PCR) (Zhao et al., 2017b).

\subsection{Indel loci detection and genotyping}

Based on the whole-genome sequencing of Chinese indigenous cattle in our lab, seven possible indel loci were found in cattle MSRB3 gene and their PCR primers were designed by Primer Premier 5.0 (Premier Biosoft International, USA) (Table 1) (Chen et al., 2018). PCR amplification was performed in a $20 \mu \mathrm{L}$ volume system: $1 \mu \mathrm{L}$ DNA $\left(50 \mathrm{ng}_{\mu \mathrm{L}^{-1}}\right)$, $10 \mu \mathrm{L} 2 \times$ PCR mix (Beijing ComWin Biotech Co., Ltd.,

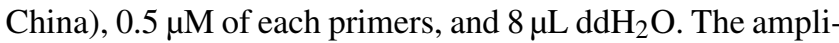
fication conditions of PCR were listed as follows: denaturing at $95^{\circ} \mathrm{C}$ for $5 \mathrm{~min}$ followed by 35 cycles of $95^{\circ} \mathrm{C}$ for $30 \mathrm{~s}$, annealing for $30 \mathrm{~s}$, and extending at $72{ }^{\circ} \mathrm{C}$ for $30 \mathrm{~s}$ followed by final extension at $72^{\circ} \mathrm{C}$ for $10 \mathrm{~min}$. PCR products were sequenced directly (Shanghai Sangon Biotech Co., Ltd., P. R. China) to identify potential mutation sites (Zhao et al., 2018). For the confirmed indel loci, every sample was amplified by the aforementioned PCR system and genotyped by $10 \%$ PAGE (polyacrylamide gel electrophoresis) (Pu et al., 2014).

\subsection{Statistical analyses}

The sequences were contrasted and analyzed by Bioedit software (UK). Hardy-Weinberg equilibrium (HWE), homozygosity (Ho), heterozygosity (He), effective allele numbers $(\mathrm{Ne})$, and polymorphism information content (PIC) were calculated and analyzed using the MSRcall website (http: //www.msrcall.com/, last access: 25 August 2018), which was based on Nei's method (Koehl et al., 2018; Yeh et al., 1999; Botstein et al., 1980; Nei, 1973). Distribution differences for genotypic and allelic frequencies among different breeds were analyzed using the $\chi^{2}$ test implemented in SPSS (version 18.0; IBM Corp., Armonk, NY, USA). Linkage disequilibrium was performed by the SHEsis online platform (http://analysis.bio-X.cn, last access: 25 August 2018) (Li et al., 2009). SPSS software (version 18.0) (International Business Machines, US) was used to calculate the associations between different genotypes and growth traits; the structure of the model is $Y_{i j k}=\mu+G_{i}+B_{j}+e_{i j k}$, where $Y_{i j k}$ is the phenotypic observations, $\mu$ is the mean of the phenotypic observations, $G_{i}$ is the effect of genotype of the $j$ th observation, $B_{j}$ is the effect of breed in the $i$ th observation, and $e_{i j k}$ is the residual effect (Liu et al., 2016; Shi et al., 2016).

\section{Results}

\subsection{Variant screening and genotyping of bovine MSRB3 gene}

In our previous study, four indel loci (P1, P2, P6, and P7) were identified from seven potential variations in bovine MSRB3 gene. Respectively, P1 was a 30 bp deletion (NC_007303.6 g.48797977-48798006 del GGGAGTAGTTACTGACTGAAGGAAAACATG); P2 was a 18 bp insertion (NC_007303.6 g.48816764-48816765 ins TTCTTTTGGCAACTGCAG); P6 was a 22 bp deletion (NC_007303.6 g.48896074-48896095 del TTTTTCTTTGTCTGGTACACTT); P7 was a $37 \mathrm{bp}$ insertion (NC_007303.6 
Table 1. Primers designed for detecting indels of MSRB3 gene in cattle.

\begin{tabular}{llrll}
\hline Loci & Primer sequences $\left(5^{\prime}-3^{\prime}\right)$ & $T \mathrm{~m}\left({ }^{\circ} \mathrm{C}\right)$ & Product size & Notes \\
\hline P1 & $\begin{array}{l}\text { F: ACGCACTGTATGATTCCA } \\
\text { R: ATAGGCCAAGATAGAGGC }\end{array}$ & 54 & $161 \mathrm{bp}$ & $\begin{array}{l}\text { Pool DNA sequencing } \\
\text { or indel genotyping }\end{array}$ \\
\hline P2 & $\begin{array}{l}\text { F: ATACAGGAAAGACAAAGAGGGG } \\
\text { R: GAGAATGGATGCTGGATGATAG }\end{array}$ & 50 & $284 \mathrm{bp}$ & $\begin{array}{l}\text { Pool DNA sequencing } \\
\text { or indel genotyping }\end{array}$ \\
\hline P3 & $\begin{array}{l}\text { F: GGCATATTACTCACTCAACA } \\
\text { R: CACAAACCAGCTTAGACTTT }\end{array}$ & 50 & $402 \mathrm{bp}$ & Pool DNA sequencing \\
\hline P4 & $\begin{array}{l}\text { F: GATCCCTGACTGGAGAACTAG } \\
\text { R: CACTGTGACCCAGACCCT }\end{array}$ & 52 & $292 \mathrm{bp}$ & Pool DNA sequencing \\
\hline P5 & $\begin{array}{l}\text { F: GTGAAGCCCTAAATGAGA } \\
\text { R: GTTGTATGGAATGGGAGTA }\end{array}$ & 50 & $223 \mathrm{bp}$ & Pool DNA sequencing \\
\hline P6 & $\begin{array}{l}\text { F: GCATAAGAAAGCCAACCT } \\
\text { R: CAGCCTCATCATCTCATCCA }\end{array}$ & 54 & $221 \mathrm{bp}$ & $\begin{array}{l}\text { Pool DNA sequencing } \\
\text { or indel genotyping }\end{array}$ \\
\hline P7 & $\begin{array}{l}\text { F: GAGCCCTAATGGATAAAA } \\
\text { R: AGTGTTGAAGTTGCCTGT }\end{array}$ & 51 & $332 \mathrm{bp}$ & $\begin{array}{l}\text { Pool DNA sequencing/ } \\
\text { indel genotyping }\end{array}$ \\
\hline
\end{tabular}

Notes: indel, insertions and deletions; MSRB3, methionine sulfoxide reductase B3; F, forward primer; R, reverse primer; $T \mathrm{~m}$, melting temperature; P1-P7, first pair to seventh pair.

g.48903091-48903092 ins AGCTGATGTATAACCTCCATAACTTGCTTTCCCCCCT); and no variation was found in P3, P4, or P5 (Fig. 1, 2). All the samples were genotyped by PCR-AFLP (amplified fragment length polymorphism). As shown in the Fig. 1a, wild type (II) showed a $161 \mathrm{bp}$ band, mutant (DD) showed a $131 \mathrm{bp}$ band, and heterozygote (ID) showed 131 and $161 \mathrm{bp}$ bands; as shown in the Fig. 1b, wild type (DD) showed a 284 bp band, mutant (II) showed a $302 \mathrm{bp}$ band, and heterozygote (ID) showed 284 and 302 bp bands; as shown in the Fig. 1c, wild type (II) showed a 221 bp band, mutant (DD) showed a 199 bp band, and heterozygote (ID) showed 199 and 221 bp bands; as shown in the Fig. 1d, wild type (DD) showed a 332 bp band, mutant (II) showed a $369 \mathrm{bp}$ band, and heterozygote (ID) showed 369 and 332 bp bands.

PCR products showed three genotypes in all of the indel loci in the cattle MSRB3 gene which were detected by $10 \%$ PAGE gel electrophoresis, including (a) the insertion and insertion type (II genotype) consisting of a $161 \mathrm{bp}$ band, deletion and deletion type (DD genotype) consisting of a $131 \mathrm{bp}$ band, and the heterozygote type (ID genotype) that showed 161 and 131 bp bands in MSRB3-1 locus; (b) the insertion and insertion type (II genotype) consisting of a $302 \mathrm{bp}$ band, deletion and deletion type (DD genotype) consisting of a $284 \mathrm{bp}$ band, and the heterozygote type (ID genotype) that showed 302 and 284 bp bands in MSRB3-2 locus; (c) the insertion and insertion type (II genotype) consisting of a 221 bp band, deletion and deletion type (DD genotype) consisting of a $199 \mathrm{bp}$ band, and the heterozygote type (ID genotype) showed 221 and 199 bp bands in MSRB3-6 locus; and (d) the insertion and insertion type (II genotype) consisting of a $369 \mathrm{bp}$ band, deletion and deletion types (DD genotype) consisting of a $332 \mathrm{bp}$ band, and the heterozygote type (ID genotype) that showed 369 and 332 bp bands in MSRB3-7 locus.

\subsection{Population genetic variability analysis in four cattle breeds}

Genetic diversity is the prerequisite for species survival, adaptation, and evolution, and is also important for improving the performance of meat production. Genotype frequency, allele frequency, and genetic parameters were estimated and shown in Table 2. For P1 locus, the breeds of QC, NY, and JX belonged to a low polymorphic locus $(0<\mathrm{PIC}<0.25)$ and $\mathrm{LX}$ belonged to a moderate polymorphic locus $(0.25<\mathrm{PIC}<0.50)$. All of them were in Hardy-Weinberg equilibrium (HWE) $(P>0.05)$. Homozygosity (Ho) ranged from 0.632 to 0.854 . Heterozygosity (He) ranged from 0.146 to 0.368 and effective allele numbers (Ne) were between 1.171 and 1.582 . The I allele (0.757$0.921)$ was higher than D allele $(0.079-0.243)$ in all four breeds. For P2 locus, LX and NY were intermediately polymorphic because they possessed moderate genetic diversity $(0.25<\mathrm{PIC}<0.50)$ and $\mathrm{QC}$ and JX belonged to low polymorphic locus $(0<\mathrm{PIC}<0.25)$. All of them were in HardyWeinberg equilibrium (HWE) except NY $(P>0.05)$. Homozygosity (Ho) ranged from 0.600 to 0.864 . Heterozygosity $(\mathrm{He})$ ranged from 0.136 to 0.400 and effective allele numbers $(\mathrm{Ne})$ were between 1.158 and 1.668 . The I allele $(0.073-$ $0.277)$ was lower than D allele $(0.723-0.927)$ in all four breeds. For P6 locus, LX and QC population deviated from the Hardy-Weinberg equilibrium among the four popula- 


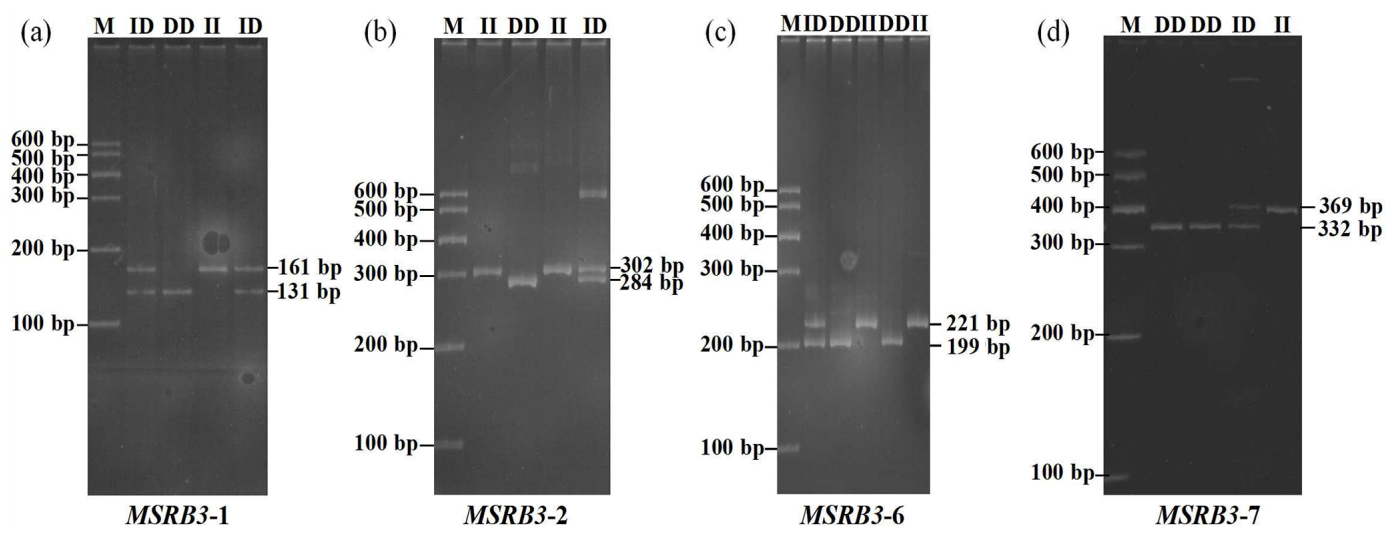

Figure 1. The agarose gel electrophoresis patterns of four indel loci in cattle MSRB3 gene.

Table 2. Genotypes, allele and population genetic information of four indel loci in MSRB3 gene.

\begin{tabular}{|c|c|c|c|c|c|c|c|c|c|c|c|c|}
\hline \multirow[t]{2}{*}{ Loci } & \multirow[t]{2}{*}{ Breeds } & \multirow{2}{*}{$\begin{array}{r}\text { Sizes } \\
N\end{array}$} & \multicolumn{3}{|c|}{$\begin{array}{l}\text { Genotypic } \\
\text { frequencies }\end{array}$} & \multicolumn{2}{|c|}{$\begin{array}{c}\text { Allelic } \\
\text { frequencies }\end{array}$} & \multirow{2}{*}{$\begin{array}{c}\text { HWE } \\
P \text { values }\end{array}$} & \multicolumn{4}{|c|}{ Population parameters } \\
\hline & & & II & ID & DD & I & $\mathrm{D}$ & & Ho & $\mathrm{He}$ & $\mathrm{Ne}$ & PIC \\
\hline \multirow{4}{*}{ P1 } & LX & 107 & 59 & 44 & 4 & 0.757 & 0.243 & $P>0.05$ & 0.632 & 0.368 & 1.582 & 0.300 \\
\hline & QC & 284 & 240 & 43 & 1 & 0.921 & 0.079 & $P>0.05$ & 0.854 & 0.146 & 1.171 & 0.135 \\
\hline & NY & 128 & 85 & 38 & 5 & 0.812 & 0.188 & $P>0.05$ & 0.854 & 0.146 & 1.171 & 0.135 \\
\hline & JX & 139 & 107 & 29 & 3 & 0.874 & 0.126 & $P>0.05$ & 0.780 & 0.220 & 1.282 & 0.196 \\
\hline \multirow{4}{*}{$\mathrm{P} 2$} & LX & 112 & 5 & 52 & 55 & 0.277 & 0.723 & $P>0.05$ & 0.600 & 0.400 & 1.668 & 0.320 \\
\hline & QC & 286 & 2 & 38 & 246 & 0.073 & 0.927 & $P>0.05$ & 0.864 & 0.136 & 1.158 & 0.127 \\
\hline & NY & 130 & 2 & 53 & 75 & 0.219 & 0.781 & $P<0.05$ & 0.658 & 0.342 & 1.521 & 0.284 \\
\hline & JX & 137 & 2 & 33 & 102 & 0.135 & 0.865 & $P>0.05$ & 0.766 & 0.234 & 1.305 & 0.206 \\
\hline \multirow{4}{*}{ P6 } & LX & 110 & 69 & 41 & 0 & 0.814 & 0.186 & $P<0.05$ & 0.697 & 0.303 & 1.435 & 0.257 \\
\hline & QC & 273 & 142 & 90 & 41 & 0.685 & 0.315 & $P<0.05$ & 0.568 & 0.432 & 1.759 & 0.338 \\
\hline & NY & 113 & 66 & 41 & 6 & 0.765 & 0.235 & $P>0.05$ & 0.641 & 0.359 & 1.560 & 0.295 \\
\hline & JX & 138 & 106 & 29 & 3 & 0.873 & 0.127 & $P>0.05$ & 0.779 & 0.221 & 1.284 & 0.197 \\
\hline \multirow{4}{*}{ P7 } & LX & 113 & 4 & 72 & 37 & 0.354 & 0.646 & $P<0.05$ & 0.543 & 0.457 & 1.843 & 0.353 \\
\hline & QC & 274 & 3 & 27 & 244 & 0.060 & 0.940 & $P<0.05$ & 0.887 & 0.113 & 1.128 & 0.107 \\
\hline & NY & 135 & 6 & 41 & 88 & 0.196 & 0.804 & $P>0.05$ & 0.684 & 0.316 & 1.461 & 0.266 \\
\hline & JX & 138 & 0 & 72 & 66 & 0.261 & 0.739 & $P<0.05$ & 0.614 & 0.386 & 1.628 & 0.311 \\
\hline
\end{tabular}

Notes: indel, insertions and deletions; MSRB3, methionine sulfoxide reductase B3; $N$, number; HWE, Hardy-Weinberg equilibrium; Ho, homozygosity; He, heterozygosity; Ne, effective allele numbers; PIC, polymorphism information content; LX, Luxi cattle; QC, Qinchuan cattle; NY, Nanyang cattle; JX, Jiaxian Red cattle; II, insertion and insertion; ID, insertion and deletion; DD, deletion and deletion.

tions. The populations of LX, QC, and JX belonged to moderate genetic diversity $(0.25<\mathrm{PIC}<0.50)$ but the JX population belonged to low polymorphic diversity $(0<P I C<0.25)$. Homozygosity (Ho) ranged from 0.568 to 0.779 . Heterozygosity (He) ranged from 0.221 to 0.432 and effective allele numbers $(\mathrm{Ne})$ were between 1.284 and 1.759 . The I allele $(0.685-0.873)$ was higher than D allele $(0.127-0.315)$ in all four breeds. For P7, LX, QC, and JX, populations deviated from the Hardy-Weinberg equilibrium, except the NY population. The populations of LX, NY, and JX belonged to moderate genetic diversity $(0.25<\mathrm{PIC}<0.50)$ but the $\mathrm{QC}$ population belonged to low polymorphic diversity $(0<\mathrm{PIC}<0.25)$. Homozygosity (Ho) ranged from 0.543 to 0.887 . Heterozy- gosity (He) ranged from 0.113 to 0.457 and effective allele numbers $(\mathrm{Ne})$ were between 1.128 and 1.843 . The I allele $(0.060-0.354)$ was lower than D allele $(0.646-0.940)$ in all four breeds. The $\chi^{2}$ test showed that there was no significant difference in the genotypic frequencies between LX and NY (P1 locus), NY and JX (P1 locus), or LX and NY (P2, P6 loci) (Table 3). In P1 locus, between QC with JX, and in P6 locus, NY with LX populations, the genotypic frequencies were significantly different, and others were extremely and significantly different. Allele frequencies were significantly different among different breeds in four loci (Table 3 ). 
Table 3. The $\chi^{2}$ test of different breeds on four indels of cattle MSRB3 gene.

\begin{tabular}{|c|c|c|c|c|c|c|}
\hline Loci & Types & Breeds & LX & QC & NY & $\mathrm{JX}$ \\
\hline \multirow[t]{2}{*}{ P1 } & $\begin{array}{l}\text { Genotypic } \\
\text { frequencies }\end{array}$ & $\begin{array}{l}\text { LX } \\
\text { QC } \\
\text { NY } \\
\text { JX }\end{array}$ & $\begin{array}{r}- \\
(p<0.01) \\
(p>0.05) \\
(p<0.01)\end{array}$ & $\begin{array}{r}\chi^{2}=39.31 \\
- \\
(p<0.01) \\
(p<0.05)\end{array}$ & $\begin{array}{r}\chi^{2}=3.40 \\
\chi^{2}=20.81 \\
- \\
(p>0.05)\end{array}$ & $\begin{array}{r}\chi^{2}=13.16 \\
\chi^{2}=5.66 \\
\chi^{2}=3.78 \\
-\end{array}$ \\
\hline & $\begin{array}{l}\text { Allelic } \\
\text { frequencies }\end{array}$ & $\begin{array}{l}\text { LX } \\
\text { QC } \\
\text { NY } \\
\text { JX }\end{array}$ & $\begin{array}{r}- \\
(p<0.01) \\
(p<0.01) \\
(p<0.01)\end{array}$ & $\begin{array}{r}\chi^{2}=33.85 \\
- \\
\quad(p<0.01) \\
(p<0.01)\end{array}$ & $\begin{array}{r}\chi^{2}=8.95 \\
\chi^{2}=51.35 \\
- \\
(p<0.01)\end{array}$ & $\begin{array}{r}\chi^{2}=45.49 \\
\chi^{2}=12.01 \\
\chi^{2}=14.52\end{array}$ \\
\hline \multirow[t]{2}{*}{ P2 } & $\begin{array}{l}\text { Genotypic } \\
\text { frequencies }\end{array}$ & $\begin{array}{l}\mathrm{LX} \\
\mathrm{QC} \\
\mathrm{NY} \\
\mathrm{JX}\end{array}$ & $\begin{array}{r}- \\
(p<0.01) \\
(p>0.05) \\
(p<0.01)\end{array}$ & $\begin{array}{r}\chi^{2}=60.07 \\
\quad(p<0.01) \\
(p<0.01)\end{array}$ & $\begin{array}{r}\chi^{2}=3.05 \\
\chi^{2}=40.80 \\
- \\
(p<0.01)\end{array}$ & $\begin{array}{r}\chi^{2}=17.27 \\
\chi^{2}=8.51 \\
\chi^{2}=8.59 \\
-\end{array}$ \\
\hline & $\begin{array}{l}\text { Allelic } \\
\text { frequencies }\end{array}$ & $\begin{array}{l}\text { LX } \\
\text { QC } \\
\text { NY } \\
\text { JX }\end{array}$ & $\begin{array}{r}- \\
(p<0.01) \\
(p<0.01) \\
(p<0.01)\end{array}$ & $\begin{array}{r}\chi^{2}=144.12 \\
- \\
\quad(p<0.01) \\
(p<0.01)\end{array}$ & $\begin{array}{r}\chi^{2}=91.38 \\
\chi^{2}=389.71 \\
- \\
(p<0.01)\end{array}$ & $\begin{array}{r}\chi^{2}=61.64 \\
\chi^{2}=20.63 \\
\chi^{2}=257.34 \\
-\end{array}$ \\
\hline \multirow{2}{*}{ P6 } & $\begin{array}{l}\text { Genotypic } \\
\text { frequencies }\end{array}$ & $\begin{array}{l}\text { LX } \\
\text { QC } \\
\text { NY } \\
\text { JX }\end{array}$ & $\begin{array}{r}- \\
(p<0.01) \\
(p<0.05) \\
(p<0.01)\end{array}$ & $\begin{array}{r}\chi^{2}=18.58 \\
- \\
\quad(p<0.01) \\
(p<0.01)\end{array}$ & $\begin{array}{r}\chi^{2}=6.03 \\
\chi^{2}=7.05 \\
- \\
(p<0.01)\end{array}$ & $\begin{array}{r}\chi^{2}=9.84 \\
\chi^{2}=27.99 \\
\chi^{2}=9.97\end{array}$ \\
\hline & $\begin{array}{l}\text { Allelic } \\
\text { frequencies }\end{array}$ & $\begin{array}{l}\text { LX } \\
\text { QC } \\
\text { NY } \\
\text { JX }\end{array}$ & $\begin{array}{r}- \\
(p<0.01) \\
(p<0.01) \\
(p<0.01)\end{array}$ & $\begin{array}{r}\chi^{2}=44.32 \\
- \\
\quad(p<0.01) \\
(p<0.01)\end{array}$ & $\begin{array}{r}\chi^{2}=7.22 \\
\chi^{2}=16.05 \\
- \\
(p<0.01)\end{array}$ & $\begin{array}{r}\chi^{2}=13.18 \\
\chi^{2}=102.65 \\
\chi^{2}=39.34 \\
-\end{array}$ \\
\hline \multirow{2}{*}{ P7 } & $\begin{array}{l}\text { Genotypic } \\
\text { frequencies }\end{array}$ & $\begin{array}{l}\mathrm{LX} \\
\mathrm{QC} \\
\mathrm{NY} \\
\mathrm{JX}\end{array}$ & $\begin{array}{r}- \\
(p<0.01) \\
(p<0.01) \\
(p<0.01)\end{array}$ & $\begin{array}{r}\chi^{2}=128.31 \\
- \\
\quad(p<0.01) \\
(p<0.01)\end{array}$ & $\begin{array}{r}\chi^{2}=27.98 \\
\chi^{2}=33.85 \\
- \\
(p<0.01)\end{array}$ & $\begin{array}{r}\chi^{2}=9.77 \\
\chi^{2}=90.64 \\
\chi^{2}=9.97 \\
-\end{array}$ \\
\hline & $\begin{array}{l}\text { Allelic } \\
\text { frequencies }\end{array}$ & $\begin{array}{l}\text { LX } \\
\text { QC } \\
\text { NY } \\
\text { JX }\end{array}$ & $\begin{array}{r}- \\
(p<0.01) \\
(p<0.01) \\
(p<0.01)\end{array}$ & $\begin{array}{r}\chi^{2}=263.28 \\
- \\
\quad(p<0.01) \\
\quad(p<0.01)\end{array}$ & $\begin{array}{r}\chi^{2}=62.61 \\
\chi^{2}=82.86 \\
- \\
(p<0.01)\end{array}$ & $\begin{array}{r}\chi^{2}=20.31 \\
\chi^{2}=149.92 \\
\chi^{2}=11.98\end{array}$ \\
\hline
\end{tabular}

Notes: indel, insertions and deletions; MSRB3, methionine sulfoxide reductase B3; LX, Luxi cattle; QC, Qinchuan cattle; NY, Nanyang cattle; JX, Jiaxian Red cattle.

\subsection{Haplotype analysis and linkage disequilibrium}

Sixteen haplotypes were analyzed in all four breeds. The frequencies are listed in Table 4, only the frequency of "P1 ${ }^{\mathrm{I}}{ }^{\mathrm{P}} 2^{\mathrm{D}} \mathrm{P} 6{ }^{\mathrm{I}}{ }^{\mathrm{P}} 7^{\mathrm{D}}$ " and " $\mathrm{P} 1^{\mathrm{I}}{ }^{\mathrm{P} 2}{ }^{\mathrm{D}} \mathrm{P}^{\mathrm{D}}{ }^{\mathrm{P}} 7^{\mathrm{D}}$ " haplotypes were all $3 \%$ higher than others in four cattle breeds. The results of linkage disequilibrium of four loci in all four breeds are shown in Fig. 3. Between the P1 and P2, P1 and P4, and P2 and $\mathrm{P} 4$ pairs, there is a strong linkage in both QC and NY. In the JX population, between $\mathrm{P} 1$ and $\mathrm{P} 2$, there is also a strong linkage.

\subsection{Association analysis between indels in MSRB3 gene and growth traits}

Association analysis, shown in Table 5, revealed that loci of P1, P6, and P7 had a significant effect on growth traits. The chest circumference of DD genotype at P1 locus was significantly longer than ID genotype in the NY population $(P<0.05)$. As for P6 locus, significant differences were found in chest circumference, hip height, and body weight in QC cattle $(P<0.01)$; the II genotype or DD genotype of body length index and chest circumference index showed better performance in the QC population $(p<0.05)$. As for P6 locus, II genotype benefited hucklebone width of the LX pop- 
Table 4. Haplotypes and their frequencies of four indels in four Chinese cattle breeds.

\begin{tabular}{|c|c|c|c|c|c|}
\hline Haplotype & Hap. seq. & $\begin{array}{l}\text { LX number } \\
\text { (frequency) }\end{array}$ & $\begin{array}{l}\text { QC number } \\
\text { (frequency) }\end{array}$ & $\begin{array}{l}\text { NY number } \\
\text { (frequency) }\end{array}$ & $\begin{array}{l}\text { JX number } \\
\text { (frequency) }\end{array}$ \\
\hline Hap 1 & $\mathrm{P} 1^{\mathrm{I}} \mathrm{P} 2^{\mathrm{I}} \mathrm{P} 6^{\mathrm{I}} \mathrm{P} 7^{\mathrm{I}}$ & $13.76(0.066)^{*}$ & - & $4.66(0.019)$ & $4.56(0.017)$ \\
\hline Hap 2 & $\mathrm{P} 1^{\mathrm{I}} \mathrm{P} 2^{\mathrm{I}} \mathrm{P} 6^{\mathrm{I}} \mathrm{P} 7^{\mathrm{D}}$ & $8.89(0.043)$ & $8.46(0.017)$ & $9.82(0.041)$ & $1.23(0.005)$ \\
\hline Hap 3 & $\mathrm{P} 1^{\mathrm{I}} \mathrm{P} 2^{\mathrm{I}} \mathrm{P} 6^{\mathrm{D}} \mathrm{P} 7^{\mathrm{I}}$ & $0.13(0.001)$ & - & $0.39(0.002)$ & $2.38(0.009)$ \\
\hline Hap 4 & $\mathrm{P} 1^{\mathrm{I}} \mathrm{P} 2^{\mathrm{D}} \mathrm{P} 6^{\mathrm{I}} \mathrm{P} 7^{\mathrm{I}}$ & $23.37(0.112)^{*}$ & $3.61(0.007)$ & $4.27(0.018)$ & $26.84(0.100)^{*}$ \\
\hline Hap 5 & $\mathrm{P} 1^{\mathrm{I}} \mathrm{P} 2^{\mathrm{D}} \mathrm{P} 6^{\mathrm{I}} \mathrm{P} 7^{\mathrm{D}}$ & $73.25(0.352)^{*}$ & $302.82(0.594)^{*}$ & $129.72(0.541)^{*}$ & $67.72(0.626)^{*}$ \\
\hline Hap 6 & $\mathrm{P} 1^{\mathrm{I}} \mathrm{P} 2^{\mathrm{D}} \mathrm{P} 6^{\mathrm{D}} \mathrm{P} 7^{\mathrm{I}}$ & $4.97(0.024)$ & $1.45(0.003)$ & - & $7.84(0.029)$ \\
\hline Hap 7 & $\mathrm{P} 1^{\mathrm{I}} \mathrm{P} 2^{\mathrm{I}} \mathrm{P} 6^{\mathrm{D}} \mathrm{P} 7^{\mathrm{D}}$ & - & $4.85(0.010)$ & $3.31(0.014)$ & - \\
\hline Hap 8 & $\mathrm{P} 1^{\mathrm{I}} \mathrm{P} 2^{\mathrm{D}} \mathrm{P}^{\mathrm{D}} \mathrm{P} 7^{\mathrm{D}}$ & $33.63(0.162)^{*}$ & $149.81(0.294)^{*}$ & $1.99(0.004)$ & $23.43(0.087)^{*}$ \\
\hline Hap 9 & $\mathrm{P}^{\mathrm{D}} \mathrm{P} 2^{\mathrm{I}} \mathrm{P} 6^{\mathrm{I}} \mathrm{P} 7^{\mathrm{I}}$ & $24.76(0.119)^{*}$ & $19.08(0.037)^{*}$ & $27.03(0.113)^{*}$ & $23.18\left(0.086^{*}\right.$ \\
\hline Hap 10 & $\mathrm{P} 1^{\mathrm{D}} \mathrm{P} 2^{\mathrm{I}} \mathrm{P} 6^{\mathrm{I}} \mathrm{P} 7^{\mathrm{D}}$ & $9.46(0.045)$ & $0.01(0.000)$ & $4.43(0.018)$ & $2.84(0.011)$ \\
\hline Hap 11 & $\mathrm{P} 1^{\mathrm{D}} \mathrm{P} 2^{\mathrm{I}} \mathrm{P} 6^{\mathrm{D}} \mathrm{P} 7^{\mathrm{I}}$ & - & $3.87(0.008)$ & - & $0.05(0.000)$ \\
\hline Hap 12 & ${ }_{\mathrm{P}}{ }^{\mathrm{D}} \mathrm{P} 2^{\mathrm{I}} \mathrm{P}^{\mathrm{D}}{ }^{\mathrm{P}} 7^{\mathrm{D}}$ & - & $0.73(0.001)$ & $0.35(0.001)$ & $0.75(0.003)$ \\
\hline Hap 13 & $\mathrm{P} 1^{\mathrm{D}} \mathrm{P} 2^{\mathrm{D}} \mathrm{P} 6^{\mathrm{I}} \mathrm{P} 7^{\mathrm{I}}$ & $6.76(0.033)$ & $1.99(0.004)$ & $5.65(0.024)$ & $1.61(0.006)$ \\
\hline Hap 14 & $\mathrm{P}^{\mathrm{D}} \mathrm{P} 2^{\mathrm{D}} \mathrm{P}^{\mathrm{I}} \mathrm{P}^{\mathrm{D}}$ & $8.75(0.042)$ & $10.02(0.020)$ & $5.41(0.023)$ & $5.02(0.019)$ \\
\hline Hap 15 & $\mathrm{P}^{\mathrm{D}} \mathrm{P}^{\mathrm{D}} \mathrm{P}^{\mathrm{D}}{ }_{\mathrm{P}}{ }^{\mathrm{I}}$ & $0.24(0.001)$ & - & - & $0.54(0.002)$ \\
\hline Hap 16 & ${ }^{P} 1^{\mathrm{D}} \mathrm{P} 2^{\mathrm{D}} \mathrm{P}^{\mathrm{D}} \mathrm{P}^{\mathrm{D}}$ & $0.03(0.000)$ & $3.29(0.006)$ & $1.12(0.005)$ & - \\
\hline
\end{tabular}

Notes: indel, insertions and deletions; hap, haplotype; hap. seq., haplotype sequence; LX, Luxi cattle; QC, Qinchuan cattle; NY, Nanyang cattle; JX, Jiaxian Red cattle; I, insertion; D, deletion; * means $p>0.03$.

Table 5. Three novel indels in MSRB3 gene and their associations with growth traits.

\begin{tabular}{|c|c|c|c|c|c|c|}
\hline \multirow[t]{2}{*}{ Loci } & \multirow[t]{2}{*}{ Breeds } & \multirow[t]{2}{*}{ Growth traits } & \multicolumn{4}{|c|}{ Observed genotypes $\left(\mathrm{LSM}^{\mathrm{a}} \pm \mathrm{SE}\right)$} \\
\hline & & & II & ID & DD & $P$ values \\
\hline P1 & NY & Body length $(\mathrm{cm})$ & $\begin{array}{r}139.11 \pm 10.36^{\mathrm{ab}} \\
(n=85)\end{array}$ & $\begin{array}{r}138.13 \pm 12.09^{\mathrm{b}} \\
(n=38)\end{array}$ & $\begin{array}{r}152.20 \pm 13.23^{\mathrm{a}} \\
(n=5)\end{array}$ & 0.05 \\
\hline \multirow{5}{*}{ P6 } & \multirow{5}{*}{ QC } & Chest circumference $(\mathrm{cm})$ & $\begin{array}{r}192.21 \pm 1.36^{\mathrm{a}} \\
(n=87)\end{array}$ & $\begin{array}{r}183.08 \pm 2.52^{\mathrm{b}} \\
(n=52)\end{array}$ & $\begin{array}{r}189.54 \pm 6.13^{\mathrm{ab}} \\
(n=12)\end{array}$ & 0.00 \\
\hline & & Hip height $(\mathrm{cm})$ & $\begin{array}{r}49.81 \pm 0.47^{\mathrm{a}} \\
(n=77)\end{array}$ & $\begin{array}{r}46.82 \pm 0.67^{\mathrm{b}} \\
(n=50)\end{array}$ & $\begin{array}{r}46.50 \pm 4.67^{\mathrm{b}} \\
(n=11)\end{array}$ & 0.01 \\
\hline & & Body weight $(\mathrm{kg})$ & $\begin{array}{r}519.50 \pm 9.31^{\mathrm{a}} \\
(n=87)\end{array}$ & $\begin{array}{r}466.80 \pm 14.43^{\mathrm{b}} \\
(n=52)\end{array}$ & $\begin{array}{r}504.11 \pm 43.90^{\mathrm{ab}} \\
(n=12)\end{array}$ & 0.01 \\
\hline & & Chest length index & $\begin{array}{r}127.69 \pm 0.90^{\mathrm{ab}} \\
(n=86)\end{array}$ & $\begin{array}{r}122.63 \pm 1.97^{\mathrm{b}} \\
(n=53)\end{array}$ & $\begin{array}{r}127.83 \pm 2.51^{\mathrm{a}} \\
(n=12)\end{array}$ & 0.03 \\
\hline & & Chest circumference index & $\begin{array}{r}148.70 \pm 0.82^{\mathrm{a}} \\
(n=87)\end{array}$ & $\begin{array}{r}144.16 \pm 1.63^{\mathrm{b}} \\
(n=52)\end{array}$ & $\begin{array}{r}147.41 \pm 3.12^{\mathrm{ab}} \\
(n=12)\end{array}$ & 0.03 \\
\hline P7 & LX & Hucklebone width $(\mathrm{cm})$ & $\begin{array}{r}30.50 \pm 2.90^{\mathrm{a}} \\
(n=4)\end{array}$ & $\begin{array}{r}29.38 \pm 0.37^{b} \\
(n=72)\end{array}$ & $\begin{array}{r}27.80 \pm 0.42^{\mathrm{b}} \\
(n=37)\end{array}$ & 0.03 \\
\hline
\end{tabular}

Notes: indel, insertions and deletions; MSRB3, methionine sulfoxide reductase B3; LSM, least squares technique, SE, standard error; LX, Luxi cattle; QC, Qinchuan cattle; NY, Nanyang cattle; II, insertion and insertion; ID, insertion and deletion; DD, deletion and deletion; ${ }^{\mathrm{a}, \mathrm{b}}$ means $P<0.05$.

ulation. The linkage disequilibrium showed that P1 and P7 were demonstrated a strong linkage in QC cattle and NY cattle $\left(r^{2}>0.33\right)$ (Fig. 3).

\section{Discussion}

In previous studies, the function of MSRB3 is related to ear morphology, auditory system, cell proliferation, and apoptosis (Kwak et al., 2017b; Lee et al., 2014). The results of GWASs have shown that MSRB3 is involved in regulation of ossification and adipose tissue development in cattle (Saatchi 

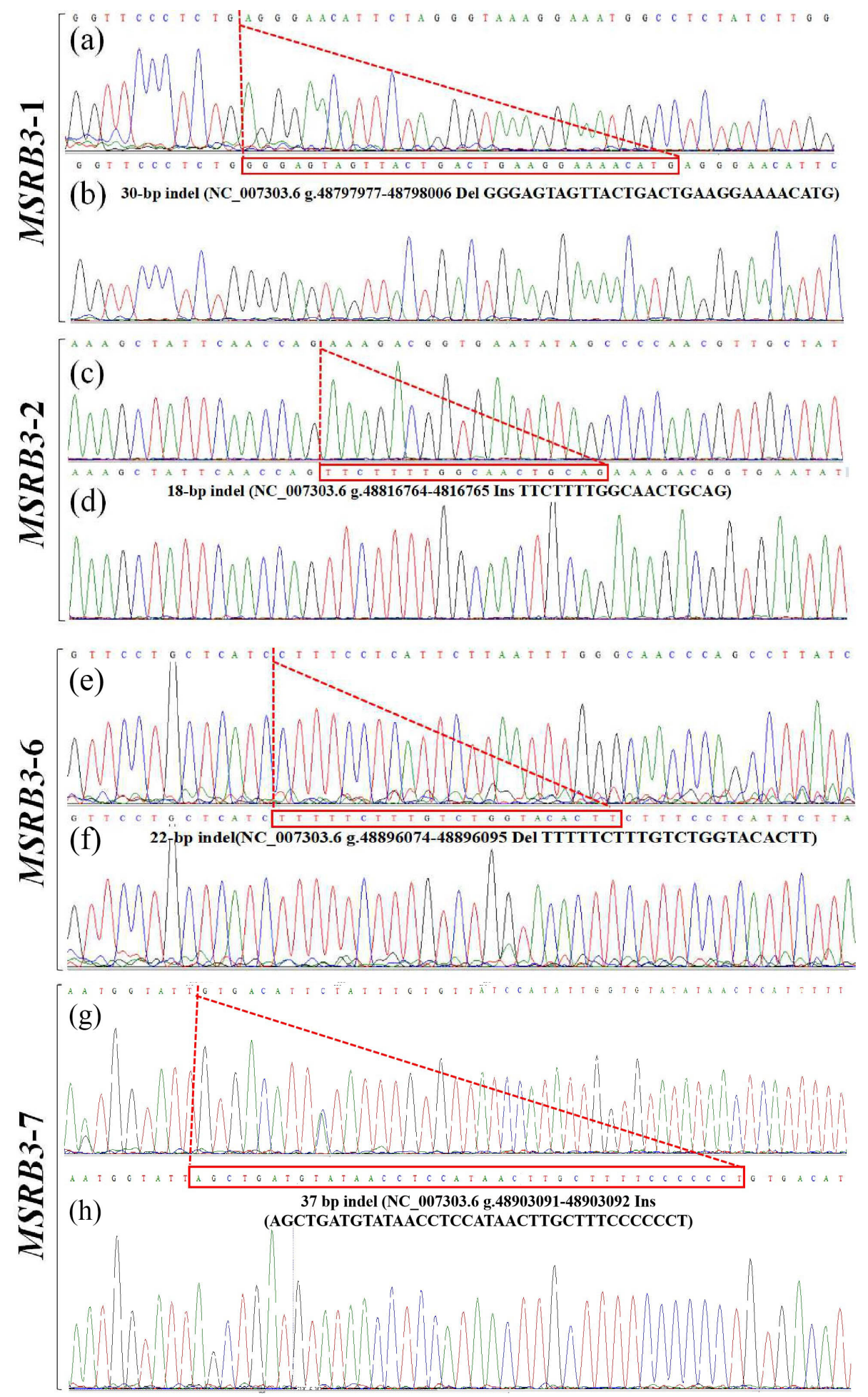

Figure 2. Sequencing maps of four indel loci in cattle MSRB3 gene. (a) Homozygotic deletion and deletion type (ID) of MSRB3-1 locus; (b) homozygotic insertion and insertion type (II) of MSRB3-1 locus, the sequence with a dashed red line boundary is a 30 bp deletion; (c) homozygotic deletion and deletion type (ID) of MSRB3-2 locus; (d) homozygotic insertion and insertion type (II) of MSRB3-2 locus, the sequence with a dashed red line boundary is a $18 \mathrm{bp}$ insertion; (e) homozygotic deletion and deletion type (ID) of MSRB3-6 locus; (f) homozygotic insertion and insertion type (II) of MSRB3-6 locus, the sequence with a dashed red line boundary is a 22 bp deletion; (g) homozygotic deletion and deletion type (ID) of MSRB3-7 locus; (h) homozygotic insertion and insertion type (II) of MSRB3-7 locus, the sequence with a dashed red line boundary is a $37 \mathrm{bp}$ insertion. 


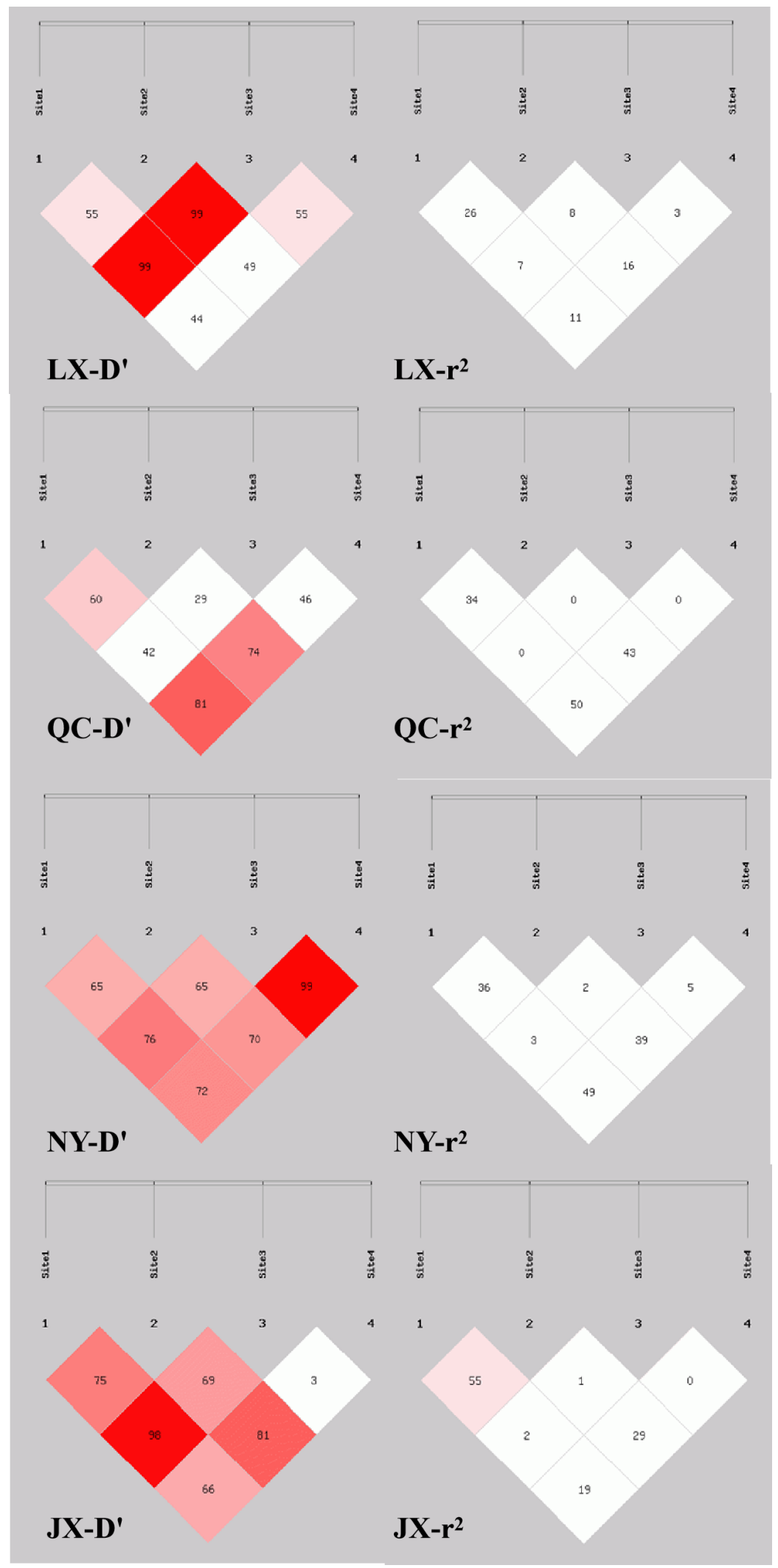

Figure 3. Linkage disequilibrium plot of four indel loci in cattle MSRB3 gene. 
et al., 2014). Some studies have shown that the knockout of $M S R B 3$ leads to a decrease in fibroblast proliferation, which could influence the function of proteins, glycosaminoglycan and glycoproteins (Lee et al., 2014). Further study revealed that inhibition of MSRB 3 could activate the expression of $P 53$, and enhance the expression level of $P 21$ and P27 subsequently (Kwak et al., 2017b). Above-mentioned studies strongly suggest that MSRB3 inhibited cell proliferation by acting on $\mathrm{p} 53, \mathrm{p} 21$, and $\mathrm{p} 27$ pathways, which suggests that MSRB3 is an important candidate gene for animal growth and development (Kwak et al., 2017b; Lee et al., 2014; Eujin et al., 2014). However, there was no study on marker-assisted selection in bovine MSRB3 gene. Therefore, we further verified the relationship between four indel loci in bovine MSRB3 gene and their growth traits.

In our study, P6 was published by NCBI (National Center of Biotechnology Information) and the remaining loci were newly discovered. The $\chi^{2}$ test showed that the P1 was in Hardy-Weinberg equilibrium but P2, P6, and P7 were not in different cattle breeds. The reason may be that artificial selection was carried out to obtain better classification characters, the genetic background of different varieties was inconsistent, or smaller sample size (Doekes et al., 2018; Zhou et al., 2018; Capellini et al., 2017). Through genetic diversity estimation, the classification of polymorphism information content (PIC) resulted in the fact that four loci were in a low polymorphism class in most of the analyzed breeds, the others were identified as having moderate polymorphism. This means that most local cattle have not been bred at these sites. On the basis of two popular measures $\left(r^{2}\right.$ and $\left.\mathrm{D}^{\prime}\right)$ performed in the linkage disequilibrium analysis, all pairs of the P1, P2, and P7 in QC and NY populations demonstrated a strong linkage. Also, a strong linkage between P1 and P2 in the JX population was shown, and others showed a weak linkage. Association analysis shows that three were related to growth traits and there is no evidence that $\mathrm{P} 2$ is related to the growth of cattle. P1 caused a $30 \mathrm{bp}$ deletion so as to affect body length in LX. Body length is an important index of meat production; therefore, it can be proved that $\mathrm{P} 1$ can be used as a novel marker for breeding (Lacerda et al., 2018; Xu et al., 2018). The deletion of P6 led to a decrease in multiple growth traits of the QC population. Growth traits of II genotype were better than ID and DD genotypes. Both P1 and P6 had harmful mutations. Association analysis of P7 resulted in the fact that individual cattle with II genotype had superior growth traits compared to individuals with ID and DD genotypes. The indels of MSRB3 included functional and nonfunctional types. Functional indels affect the gene product and growth traits, whereas nonfunctional indels may be strongly linked with functional indels of MSRB3.

In a nutshell, the indels of P1, P6, and P7 within MSRB3 gene significantly affected growth traits, meaning that $\mathrm{P} 1$, P6, and P7 could be used as DNA markers for eliminating or selecting excellent individuals in marker-assisted selection
(MAS) breeding in relation to growth traits, and MSRB3 gene could be used as a candidate gene for breeding beef cattle.

\section{Conclusions}

This research identified four indels within MSRB3 gene firstly, which were associated with notable growth traits in four Chinese indigenous cattle breeds. Meanwhile, functional indels could be utilized as promising molecular markers in early marker-assisted selection for cattle breeding after the further extensive research.

Data availability. Data are available upon request.

Author contributions. RD and MW designed the experiments; SL and GZ collected DNA samples; MW, YF, and YG performed the experiments; YH and XL collected growth data; YM and CL analyzed the experimental data; and RD and MW wrote the paper.

Competing interests. The authors declare that they have no conflict of interest.

Acknowledgements. This work was supported by the Key Research \& Development Plan of Shaanxi Province of China (General Project) (2017NY-071), the Science and Technology Plan of Yangling Demonstration Zone (2018NY-33) and the National Beef Cattle and Yak Industrial Technology System (CARS-37).

Financial support. This research has been supported by the Key Research \& Development Plan of Shaanxi Province of China (grant no. 2017NY-071), the Science and Technology Plan of Yangling Demonstration Zone (grant no. 2018NY-33), and the National Beef Cattle and Yak Industrial Technology System (grant no. CARS-37).

Review statement. This paper was edited by Steffen Maak and reviewed by Xiaomei Sun and one anonymous referee.

\section{References}

Botstein, D., White, R. L., Skolnick, M., and Davis, R. W.: Construction of a genetic linkage map in man using restriction fragment length polymorphisms, Am. J. Hum. Genet., 32, 314-331, 1980.

Capellini, T. D., Chen, H., Cao, J., Doxey, A. C., Kiapour, A. M., Schoor, M., and Kingsley, D. M.: Ancient selection for derived alleles at a GDF5 enhancer influencing human growth and osteoarthritis risk, Nat. Genet., 49, 1202-1210, 2017.

Chen, N., Cai, Y., Chen, Q., Li, R., Wang, K., Huang, Y., Hu, S., Huang, S., Zhang, H., Zheng, Z., Song, W., Ma, Z., Ma, Y., Dang, R., Zhang, Z., Xu, L., Jia, Y., Liu, S., Yue, X., Deng, 
W., Zhang, X., Sun, Z., Lan, X., Han, J., Chen, H., Bradley, D. G., Jiang, Y., and Lei, C.: Whole-genome resequencing reveals world-wide ancestry and adaptive introgression events of domesticated cattle in East Asia, Nat. Commun., 9, 2337, https://doi.org/10.1038/s41467-018-04737-0, 2018.

Doekes, H. P., Veerkamp, R. F., Bijma, P., Hiemstra, S. J., and Windig, J. J.: Trends in genome-wide and region-specific genetic diversity in the Dutch-Flemish Holstein-Friesian breeding program from 1986 to 2015, Genet. Sel. Evol., 50, 15, https://doi.org/10.1186/s12711-018-0385-y, 2018.

Eujin, L., GeunHee, K., Kranti, K., and HwaYoung, K.: Methionine sulfoxide reductase B3 deficiency inhibits cell growth through the activation of p53-p21 and p27 pathways, Arch. Biochem. Biophys., 547, 1-5, 2014.

Fan, Y., Wang, W., Ma, G., Liang, L., Shi, Q., and Tao, S.: Patterns of insertion and deletion in Mammalian genomes, Curr. Genomics, 8, 370-378, 2007.

Gui, L., Jiang, B., Zhang, Y., and Zan, L.: Sequence variants in the bovine silent information regulator 6 , their linkage and their associations with body measurements and carcass quality traits in Qinchuan cattle, Gene, 559, 16-21, 2015.

Huang, J., Zheng, Q., Wang, S., Zhang, Q., Jiang, L., Hao, R., Li, F., and Ma, Y.: A combined genotype of three SNPs in the bovine gene is related to growth performance in Chinese cattle, Arch. Anim. Breed., 60, 357-362, 2017.

Huang, J., Chen, N., Li, X., An, S., Zhao, M., Sun, T., Hao, R., and Ma, Y.: Two Novel SNPs of PPAR $\gamma$ Significantly Affect Weaning Growth Traits of Nanyang Cattle, Anim. Biotechnol, 29, 6874, 2018.

Koehl, A. J. and Long, J. C.: The contributions of admixture and genetic drift to diversity among post-contact populations in the Americas, Am. J. Phys. Anthropol., 165, 256-268, https://doi.org/10.1002/ajpa.23347, 2018.

Kwak, G. H. and Kim, H. Y.: MsrB3 deficiency induces cancer cell apoptosis through p53-independent and ER stress-dependent pathways, Arch. Biochem. Biophys., 621, 1-5, $2017 \mathrm{~b}$.

Kwak, G. H., Kim, T. H., and Kim, H. Y.: Down-regulation of MsrB3 induces cancer cell apoptosis through reactive oxygen species production and intrinsic mitochondrial pathway activation, Biochem. Biophys. Res. Commun., 483, 468-474, 2017a.

Lacerda, V. V., Campos, G. S., Roso, V. M., Souza, F. R. P., Brauner, C. C., and Boligon, A. A.: Effect of mature size and body condition of Nelore females on the reproductive performance, Theriogenology, 118, 27-33, 2018.

Lee, E., Kwak, G. H., Kamble, K., and Kim, H. Y.: Methionine sulfoxide reductase B3 deficiency inhibits cell growth through the activation of p53-p21 and p27 pathways, Arch. Biochem. Biophys., 547, 1-5, 2014.

Lee, H., Jaffe, A. E., Feinberg, J., Tryggvadottir, R., Brown, S., Montano, C., Aryee, M. J., Irizarry, R. A., Herbstman, J., Witter, F. R., Goldman, L. R., Feinberg, A. P., and Fallin, M. D.: DNA methylation shows genome-wide association of NFIX, RAPGEF2 and MSRB3 with gestational age at birth, Int. J. Epidemiol, 41, 188-199, 2012.

Li, Z., Zhang, Z., He, Z., Tang, W., Li, T., Zeng, Z., He, L., and Shi, Y.: A partition-ligation-combination-subdivision EM algorithm for haplotype inference with multiallelic markers: update of the SHEsis (http://analysis.bio-x.cn), Cell Res., 19, 519-523, 2009.
Liu, M., Li, M., Wang, S., Xu, Y., Lan, X., Li, Z., Lei, C., Yang, D., Jia, Y., and Chen, H.: Association analysis of bovine Foxa2 gene single sequence variant and haplotype combinations with growth traits in Chinese cattle, Gene, 536, 385-392, 2016.

Nei, M.: Analysis of gene diversity in subdivided populations, P. Natl. Acad. Sci. USA, 70, 3321-3323, 1973.

Pillas, D., Hoggart, C. J., Evans, D. M., O’Reilly, P. F., Sipilä, K., Lähdesmäki, R., Millwood, I. Y., Kaakinen, M., Netuveli, G., Blane, D., Charoen, P., Sovio, U., Pouta, A., Freimer, N., Hartikainen, A. L., Laitinen, J, Vaara, S., Glaser, B., Crawford, P., Timpson, N. J., Ring, S. M., Deng, G., Zhang, W., McCarthy, M. I., Deloukas, P., Peltonen, L., Elliott, P., Coin, L. J., Smith, G. D., and Jarvelin, M. R.: Genome-wide association study reveals multiple loci associated with primary tooth development during infancy, PLoS. Genet., 6, e1000856, https://doi.org/10.1371/journal.pgen.1000856, 2010.

Pu, Y., Zhang, Z., Zhou, B., Chen, P., Zhang, K., Song, Y., Gao, Q., Wang, K., Quan, Y., Xi, M., and Zhang, L.: Association of an insertion/deletion polymorphism in IL1A 3'-UTR with risk for cervical carcinoma in Chinese Han Women, Hum. Immunol., 75, 740-744, 2014.

Saatchi, M., Schnabel, R. D., Taylor, J. F., and Garrick, D. J.: Large-effect pleiotropic or closely linked QTL segregate within and across ten US cattle breeds, BMC Genomics, 15, 442, https://doi.org/10.1186/1471-2164-15-442, 2014.

Shen, X., Liu, F., Wang, Y., Wang, H., Ma, J., Xia, W., Zhang, J., Jiang, N., Sun, S., Wang, X., and Ma, D.: Down-regulation of msrb3 and destruction of normal auditory system development through hair cell apoptosis in zebrafish, Int. J. Dev. Biol., 59, 195-203, 2015.

Shi, T., Peng, W., Yan, J., Cai, H., Lan, X., Lei, C., Bai, Y., and Chen, H.: A novel $17 \mathrm{bp}$ indel in the SMAD3 gene alters transcription level, contributing to phenotypic traits in Chinese cattle, Arch. Anim. Breed., 59, 151-157, 2016.

Singh, S., Sharma, S., Arora, J. S., and Sarkhel, B. C.: Molecular and biochemical evaluation of Indian draft breeds of cattle (Bos indicus), Biochem. Genet., 49, 242-250, 2011.

Wei, C., Wang, H., Liu, G., Wu, M., Cao, J., Liu, Z., Liu, R., Zhao, F., Zhang, L., Lu, J., Liu, C., and Du, L.: Genomewide analysis reveals population structure and selection in Chinese indigenous sheep breeds, BMC Genomics, 16, 194, https://doi.org/10.1186/s12864-015-1384-9, 2015.

Xu, W., He, H., Zheng, L., Xu, J. W., Lei, C. Z., Zhang, G. M., Dang, R. H., Niu, H., Qi, X. L., Chen, H., and Huang, Y. Z.: Detection of 19 bp deletion within PLAG1 gene and its effect on growth traits in cattle, Gene, 675, 144-149, 2018.

Yeh, F. C., Yang, R., and Boyle, T.: POPGENE Version 3.1, Microsoft Window-based Free-ware for Population Genetic Analysis, University of Alberta, Edmonton, AB, Canada 1999.

Yue, B., Wu, J., Shao, S., Zhang, C., Fang, X., Bai, Y., Qi, X., and Chen, H.: Polymorphism in PLIN2 gene and its association with growth traits in Chinese native cattle, Anim. Biotechnol., 5, 1-6, https://doi.org/10.1080/10495398.2018.1555166, 2019.

Zhang, Q., Jin, Y., Jiang, F., Cheng, H., Wang, Y., Lan, X., and Song, E.: Relationship between an indel mutation within the SIRT4 gene and growth traits in Chinese cattle, Anim. Biotechnol., 28, 1-6, https://doi.org/10.1080/10495398.2018.1520716, 2019.

Zhang, W., Gao, X., Zhang, Y., Zhao, Y., Zhang, J., Jia, Y., Zhu, B., Xu, L., Zhang, L., Gao, H., Li, J., and Chen, Y.: Genome-wide 
assessment of genetic diversity and population structure insights into admixture and introgression in Chinese indigenous cattle, BMC Genet., 19, 114, https://doi.org/10.1186/s12863-018-07059, 2018.

Zhang, Z. and Gerstein, M.: Patterns of nucleotide substitution, insertion and deletion in the human genome inferred from pseudogenes, Nucleic Acids Res., 31, 5338-5348, 2003.

Zhang, L. C., Liang, J., Pu, L., Zhang, Y. B., Wang, L. G., Liu, X., Yan, H., and Wang, L. X.: mRNA and protein expression levels of four candidate genes for ear size in Erhualian and Large White pigs, Genet. Mol. Res., 16, 28407177, https://doi.org/10.4238/gmr16029252, 2017.

Zhao, H., He, S., Wang, S., Zhu, Y., Xu, H., Luo, R., Lan, X., Cai, Y., and Sun, X.: Two New Insertion/Deletion Variants of the PITX2 Gene and their Effects on Growth Traits in Sheep, Anim. Biotechnol, 29, 1-7, https://doi.org/10.1080/10495398.2017.1379415, 2017b.
Zhao, H. D., He, S., Zhu, Y. J., Cao, X., Luo, R. Y., Cai, Y., Xu, H. W., and Sun, X. Z.: A novel $29 \mathrm{bp}$ insertion/deletion (indel) variant of the LHX3 gene and its influence on growth traits in four sheep breeds of various fecundity, Arch. Anim. Breed, 60, 79-85, 2017a.

Zhao, H. D., Wu, M. L., Wang, S. H., Yu, X. H., Li, Z., Dang, R. H., and Sun, X. Z.: Identification of a novel $24 \mathrm{bp}$ insertion/deletion (indel) of the androgen receptor gene and its association with growth traits in four indigenous cattle breeds, Arch. Anim. Breed, 61, 71-78, 2018.

Zhou, Z., Li, M., Cheng, H., Fan, W., Yuan, Z., Gao, Q., Xu, Y., Guo, Z., Zhang, Y., Hu, J., Liu, H., Liu, D., Chen, W., Zheng, Z., Jiang, Y., Wen, Z., Liu, Y., Chen, H., Xie, M., Zhang, Q., Huang, W., Wang, W., Hou, S., and Jiang, Y.: An intercross population study reveals genes associated with body size and plumage color in ducks, Nat. Commun., 9, 2648, https://doi.org/10.1038/s41467-018-04868-4, 2018. 